\title{
Fuentes de información, indicadores y herramientas más usadas por gerentes de Mipyme en Cali, Colombia
}

Patricia González

González

Facultad de Ciencias de la Administración, Universidad del Valle, Cali-Colombia pagonza@univalle.edu.co

Tatiana Bermúdez Facultad de Ciencias de la Administración, Universidad del Valle, Cali-Colombia

\footnotetext{
${ }^{1}$ Micro, pequeñas y medianas empresas.
}

\section{Resumen}

Este artículo presenta los resultados de una investigación de carácter descriptivo que pretende identificar las fuentes de información, los indicadores y las herramientas gerenciales más utilizadas por gerentes de Mipyme ${ }^{1}$ en Cali, Colombia. Para tal fin se realizó una encuesta a 270 gerentes de Mipyme, cuyas empresas se encuentran inscritas en la Cámara de Comercio de ese país. Los criterios utilizados para clasificarlas como micro, pequeñas o medianas empresas fueron los que establece la Ley 590 de 2000. Se observó que los gerentes de las Mipyme principalmente se apoyan para la toma de decisiones en indicadores financieros, los cuales surgen de la combinación de indicadores tanto de rentabilidad como de liquidez y endeudamiento. Las principales fuentes de información son el Balance General, el Estado de Resultados y el Flujo de Efectivo. Se constató que en muy bajo porcentaje los gerentes encuestados consideran el Valor Económico Agregado para la toma de decisiones. En cuanto a indicadores no financieros, prevalecen los relacionados con clientes y mercado, mientras que los que miden capacitación de funcionarios, uso de tecnologías e innovación en los procesos se emplean en porcentajes muy bajos. Con relación al conocimiento y aplicación de herramientas gerenciales, un alto porcentaje de los gerentes encuestados afirmaron desconocerlas, por lo que no las implementan; sin embargo, el bajo porcentaje de gerentes que contestaron conocerlas mencionó el balanced scorecard (cuadro de mando integral) y costos $\mathrm{ABC}$; en cambio la teoría de restricciones es desconocida por ellos. 
Palabras clave: micro, pequeñas y medianas empresas (Mipyme); balanced scorecard, costos $\mathrm{ABC}$, teoría de las restricciones, indicadores financieros; indicadores no financieros.

\title{
Main sources of information, indicators and management tools used by ma- nagers of MSMEs, in Cali, Colombia
}

\begin{abstract}
This article presents the results of a descriptive type investigation seeking to identify sources of information, indicators and management tools used by managers of MSMEs ${ }^{1}$ located in Cali, Colombia. To this end, a survey was made among 270 managers of MSMEs registered in the Chamber of Commerce of said country. The criteria used for their ranking as micro, small or medium businesses were those set by the 590 Act of 2000. We could observe that MSMEs' managers rely mainly, for their decision making, on financial indicators which are the combination of profitability, liquidity and debt indicators. Main sources of information identified are: Balance Sheet, Income statement and Cash flow statement. We also confirmed that Economic Value Added is given a very low percentage of consideration by those surveyed for their decision making. In regard to non-financial indicators, those related to market and customers prevail, while those measuring staff training, technology use and process innovation are used in a very low percentage. As for management tools, a high percentage of the managers surveyed report to be unfamiliar with them and, thereby, they do not implement them. Nevertheless, those few acquainted with such tools mentioned the balanced scorecard and $\mathrm{ABC}$ costs. The theory of constraints is not familiar among those surveyed.
\end{abstract}

Keywords: micro, small and medium enterprises (MSMEs), balanced scorecard, ABC costs, theory of constraints, financial indicators, non-financial indicators.

\section{Introducción}

Dentro del marco del proyecto de investigación Una aproximación al modelo de toma de decisiones usado por los gerentes de las micro, pequeñas y medianas empresas ubicadas en la ciudad de Cali, Colombia se diseñó una encuesta que se aplicó a gerentes de Mipyme de Cali, la cual tenía entre sus principales objetivos determinar el modelo de decisión usado por los gerentes e identificar las fuentes de información e indicadores (financieros y no financieros), así como las herramientas gerenciales más usadas en la toma de decisión por parte de los gerentes de

\footnotetext{
${ }^{1}$ This term refers to micro, small and medium enterprises.
} 
estas unidades productivas. Cabe destacar que en este artículo el análisis se limita a identificar las fuentes de información, los indicadores y las herramientas gerenciales que con mayor frecuencia suelen utilizar los gerentes de las Mipyme en el proceso de toma de decisión.

El artículo se divide en las siguientes partes: la primera parte abarca la formulación del problema. En la segunda se presentan los objetivos. En la tercera se expone el marco conceptual y teórico, en el que se aborda el tema de la organización inteligente en cuanto al uso de la información; para ello se reseñan brevemente las siguientes herramientas gerenciales: balanced scorecard, costos ABC y teoría de las restricciones; además, se aborda el tema de los indicadores financieros y no financieros. En la cuarta se identifican las hipótesis. En la quinta se presentan los aspectos metodológicos considerados en la formulación de las encuestas; asimismo, se justifica el tamaño de la muestra y se presentan las limitaciones del trabajo. La sexta corresponde al análisis descriptivo de los principales resultados de la encuesta. Finalmente, en la séptima se exponen las conclusiones de la investigación.

\section{Formulación del problema de investigación}

Colombia es un país con un proceso de reindustrialización permanente, el cual ha sido provocado por la consolidación de un crecimiento económico, afectado en los últimos años por la crisis económica mundial. La mayoría de las unidades productivas son Mipyme, pues el número de ellas supera el 1.2 millones; además, son las responsables del $60 \%$ del empleo industrial.

Como consecuencia de la importancia que tienen las Mipyme en el contexto económico y social colombiano, éstas se han convertido en el foco de interés de los bancos, de empresas que ofrecen tecnología, de empresas editoras que se interesan en realizar publicaciones especializadas sobre ellas e inclusive se han creado y fortalecido programas de emprendimiento dirigidos a incentivar la creación de pequeñas y medianas empresas.

Debido al impacto económico y social que estas empresas generan en el contexto nacional es prioritario realizar estudios que permitan identificar aquellos puntos débiles que pueden comprometer su continuidad o su funcionamiento de manera eficiente y eficaz; por lo tanto, se vuelve necesario presentar soluciones o alternativas que conduzcan al fortalecimiento de las mismas. 
La forma como las empresas en general utilizan la información es un elemento que determina las ventajas competitivas entre las organizaciones. El uso estratégico de la información permite que los gerentes de las Mipymes utilicen la información para percibir los cambios del medioambiente, representen dichos cambios y a partir de las representaciones creen conocimiento y tomen decisiones. Sin embargo, es necesario aclarar que el proceso de toma de decisión no siempre es fácil debido a que, si bien es cierto las organizaciones son racionales, no lo son las personas que laboran en ellas, pues las decisiones que toman pueden estar sesgadas por factores como preferencias e intereses, entre otros. No obstante, se han creado mecanismos que facilitan la toma de decisión con los que se garantiza cierto grado de racionalidad por parte de los individuos que participan en los procesos de toma de decisión al interior de la empresa.

Entre los mecanismos que ayudan a la toma de decisión se encuentran los estados financieros básicos, los indicadores financieros y no financieros, así como las herramientas gerenciales como el balanced scorecard, la teoría de las restricciones y los costos $\mathrm{ABC}^{3}$, las cuales a partir de sus premisas y modelos de decisión orientan el actuar de los gerentes para el logro del objetivo máximo que es el de crear valor.

En ese orden de ideas, nuestra investigación pretende dar respuesta a las siguientes preguntas: ¿Utilizan los gerentes de las empresas Mipyme fuentes de información de carácter financiero y no financiero en el proceso de toma de decisión? ¿Utilizan los gerentes de las empresas Mipyme solamente indicadores de carácter financiero para la toma de decisión? ¿Cuáles son las herramientas gerenciales (de las propuestas para esta investigación) que suelen utilizar con mayor frecuencia los gerentes de la Mipyme para tomar decisiones?

\section{Objetivos}

El objetivo general de este trabajo consiste en identificar las principales fuentes de información, indicadores y herramientas gerenciales usadas por los gerentes de las Mipymes en el proceso de toma de decisión.

\footnotetext{
${ }^{3} \mathrm{~A}$ pesar de que existen otras herramientas gerenciales, las señaladas fueron seleccionadas para esta investigación.
} 
Los objetivos específicos se centran en lo siguiente: identificar las principales fuentes de información financieras y no financieras que son utilizadas por los gerentes en la toma de decisiones; conocer otros tipos de informes diferentes a los financieros, que facilitan la toma de decisión; identificar los indicadores de carácter financiero y no financiero más utilizados por los gerentes encuestados; asimismo, identificar las herramientas gerenciales (de las propuestas para esta investigación) más utilizadas por los gerentes de las Mipyme.

\section{Marco conceptual y teórico}

\section{El estado del arte de las Mipyme en Colombia}

La estructura productiva de Colombia se caracteriza por la existencia de pequeños establecimientos productivos donde el tamaño de la empresa influye en la capacidad para innovar, competir, exportar y financiarse. Los estudios recientes elaborados por la Asociación Colombiana de las Micro, Pequeñas y Medianas Empresas (ACOPI) muestran la reducida dimensión de las Mipyme en aspectos permanentes como la excesiva dependencia de sus fondos propios, la restricción al acceso del crédito bancario, la utilización más financiera que comercial de los créditos de provisión y la existencia de costos financieros elevados dada su posición objetiva de riesgo. Asimismo, existen otras deficiencias estructurales que dificultan el desarrollo de las micro, pequeñas y medianas empresas y que las limitan en el aprovechamiento de las ventajas de la globalización como son los reducidos mercados, el bajo desarrollo tecnológico, la baja calificación de la fuerza de trabajo, las limitaciones para aplicar nuevas técnicas de gestión, el precario diseño organizativo y las limitaciones para actividades exportadoras.

El desarrollo de las micro, pequeñas y la medianas empresas se sustenta, en gran medida, por la implementación de políticas estatales que permiten a estas unidades productivas fortalecer sus actividades, así como por los modelos de gestión que éstas implementan según sus objetivos y estrategias; ambas conforman el contexto tecno-productivo de este tipo de organizaciones.

En Colombia, las políticas públicas que involucran a las Mipyme se han centrado en superar las restricciones estructurales y a mejorar las condiciones por medio de la autoridad y recursos públicos. En la historia legislativa del país se pueden identificar tres hechos fundamentales en materia de leyes para el apoyo al desarrollo de las micro, pequeñas y medianas empresas: el Plan Nacional para el Desarrollo 
de la Microempresa en 1984, la Ley 590 de 2000 y la Ley 905 de 2004, esta última es la que articula la normatividad sectorial y el acceso de recursos a pequeños productores.

Además, el Consejo Nacional de Política Económica y Social (CoNPES) recomienda políticas hacia las micro, pequeñas y medianas empresas para ser puestas en marcha por el Gobierno Nacional de acuerdo con lo que se establezca en el respectivo Plan Nacional de Desarrollo. El objetivo de las recomendaciones consiste en que dichas empresas se constituyan en una fuente creciente de generación de ingresos y empleo de calidad, de tal forma que logren insertarse y posicionarse en los mercados nacionales e internacionales (Documentos ConPes 3484 y 3424).

\section{Las Mipyme en la Ciudad de Cali}

Las pymes del área metropolitana de Cali, Yumbo participan con el 11,4\% de las 24.118 que existen en Colombia. El Censo Económico de Cali, Yumbo encontró 2.738 pyme que equivalen al 5,1\% del total de empresas; sin embargo, el mayor porcentaje corresponde a las micro con una participación de $94,4 \%$; por último, se encuentran las grandes empresas con tan sólo un $0.5 \%$ de participación (DANE, Censo Económico Cali, Yumbo, 2005).

En el nivel sectorial, el área metropolitana de Cali, Yumbo presenta un comportamiento similar al nacional porque el $51 \%$ de las pyme forman parte del sector servicios y el $62 \%$ de las micro se dedican al comercio; en cuanto a empleo, las microempresas generan el $41 \%$ del empleo, las pyme el 26,6\% y las grandes empresas el 32,4\% del empleo del área metropolitana de Cali, Yumbo.

De esta manera, tanto en la ciudad de Cali como en el resto del país, las Mipyme juegan un papel fundamental en la economía por su dinamismo.

\section{La toma de decisión en las organizaciones inteligentes}

Uno de los principales aspectos por ser considerados en esta investigación es ¿cómo las organizaciones usan la información de manera estratégica? La creación y el uso de la información tienen un carácter determinante para que las organizaciones crezcan y se adapten; para ello, la teoría de dirección y organización hace énfasis en el estudio de tres campos de uso estratégico de la información: percepción, creación de conocimiento y toma de decisión. 
El ambiente en que se desarrolla una organización impacta y define de manera significativa muchas de sus características; es así que la penetración y los cambios en el mercado determinan el desempeño de la organización; asimismo, las normas fiscales, contables y comerciales definen su identidad y ámbito de influencia. El público en general y sus costumbres sociales, la mayoría de ellas plasmadas en las normas, limitan el papel que desempeña la organización y su alcance.

Las organizaciones que tienen un conocimiento previo de cómo se conforma y comporta el medioambiente tendrán una ventaja competitiva con respecto a aquellas que no lo están. La fase de percepción de cambios en el medioambiente externo tiene el inconveniente de que los mensajes que surgen de los cambios sean mal interpretados por su ambigüedad y escasez de los mismos. Por tanto, la gerencia debe descubrir los cambios más importantes, interpretarlos para tomar medidas de acción que lleven a respuestas apropiadas.

De acuerdo con Choo (1999), la meta a corto plazo en el campo de la percepción consiste en que los miembros tengan un conocimiento de lo que es la organización y de lo que está haciendo; en cambio, un objetivo de largo plazo, en este mismo campo, es garantizar que la organización se adapte y permanezca en un medioambiente dinámico. El segundo campo de uso estratégico de la información aparece cuando las organizaciones crean, organizan y procesan información, con lo cual generan nuevo conocimiento a través del aprendizaje organizacional.

Para Drucker (1993) el conocimiento, antes que el capital o la mano de obra, es el único recurso económico importante de la sociedad poscapitalista; por tanto, la gerencia debe asegurar la aplicación y ejecución del conocimiento, o sea, aplicar el conocimiento al conocimiento.

En las organizaciones actuales, la creación y el uso del conocimiento se han convertido en un asunto generador de desafíos porque muchas veces implican la superación de obstáculos que al interior de la organización existen e impiden que la creación y el uso del conocimiento se constituyan en fuentes de información creadoras de valor para la organización. Entre los obstáculos por mencionar están la falta de capacidad de la organización para identificar dónde están las fuentes de experiencia que existen al interior de la misma. Del mismo modo, las organizaciones presentan dificultades para cambiar premisas del pasado que en su momento fueron correctas, pero que en la actualidad no lo son y que al no ser revisadas y actualizadas pueden llevar a la compañía al fracaso. Para Senge, citado por Choo 
(1999), muchas organizaciones son incapaces de funcionar cabalmente como organizaciones basadas en el conocimiento porque padecen incapacidades para el aprendizaje; por ello, este autor sugiere que la organización que aprende tiene que desarrollar habilidades para el aprendizaje generativo y adaptativo.

Finalmente, el tercer campo de uso estratégico de la información se da cuando la organización busca y evalúa información con el fin de que le sirva para tomar decisiones. Teóricamente, la selección de información para fines de toma de decisión se debe realizar de una manera racional (March y Simon, citado por Choo, 1999); por consiguiente, es necesario tener información completa sobre los objetivos de la organización, las alternativas factibles, los resultados probables de las mismas y los valores de importancia que tales resultados tienen para la organización.

En la vida real, la elección racional de la información es sesgada por aspectos como forcejeo entre quienes tienen intereses en la organización, el regateo y negociación entre grupos e individuos poderosos, las limitaciones e idiosincrasias de las selecciones personales y la falta de información. No obstante, la organización debe tratar de mantener un grado de racionalidad a pesar de todos esos factores que afectan la calidad de la información, de no hacerse así, se perdería la apariencia de conducta racional y de consistencia que la organización debe transmitir con el fin de mantener la confianza al interior y la legitimidad al exterior de ella.

La toma de decisión se convierte, así, en uno de los procesos más difíciles que una organización debe enfrentar porque es un proceso complejo, enredado y esencial, donde todas las acciones que realiza una organización son el resultado de la toma de decisiones; en otras palabras, toda decisión es un compromiso para emprender una acción (Simon, 1993; March, 1976).

Indicadores financieros y no financieros en los procesos de toma de decisión

Los gerentes de las Mipyme en el proceso de toma de decisión de carácter racional y lógico cuentan con instrumentos como los indicadores que son medidas que permiten constatar el resultado de un proceso, el logro o avance en el desarrollo de una estrategia previamente establecida.

Entre los indicadores de resultado están los financieros y los no financieros que miden relación con clientes en todas sus formas. Por otro lado, existen los indicadores 
que miden desempeño y que algunos autores denominan inductores, pues son los que inducen o contribuyen con el logro de un resultado final.

Los indicadores de carácter financiero tuvieron su origen y máxima importancia en la era industrial. En esa época las empresas que tuvieran una mayor cantidad de inventarios y activos fijos gozaban de una ventaja competitiva. Dichos activos se podían medir financieramente una vez que la información que se derivaba de su uso o aplicación se podía calcular y expresar en forma de datos que conformaban los estados financieros como el Balance General y el Estado de Resultados. Es así que los gerentes comienzan a tomar decisiones a partir de calcular indicadores de carácter financiero con los datos que eran tomados de los estados financieros. En consecuencia, las empresas comienzan a medir su liquidez, rentabilidad, endeudamiento y eficiencia en el manejo de sus activos para fines de toma de decisión. Otro indicador de carácter no financiero era la información que en ese momento formaba parte de una lista, del que no se obtenía ningún provecho y que incluso ocasionaba que algunas empresas no concibieran indicadores diferentes a los ya existentes y mucho menos que éstos fueran de carácter no financiero (Kaplan y Norton, 2001).

Este panorama va cambiando debido a dos fenómenos fundamentales: los avances tecnológicos y la diversificación de los productos y servicios; éstos a su vez van marcando un cambio en el contexto de los negocios caracterizado por organizaciones con estructuras funcionales integradas, decisiones de producción provocadas por los clientes y no por presupuestos de producción, la innovación en los productos y servicios, la apertura global que rompe con la tradición de negociar al interior de las fronteras y el empoderamiento de los trabajadores.

El nuevo ambiente de los negocios trae como consecuencia que la ventaja competitiva no dependa más de los activos tangibles, sino de los intangibles. Esta situación llevó a un replanteamiento en los indicadores que se utilizarían para medir el desempeño de las organizaciones; éste ya no sólo se podía medir a través de indicadores tradicionales como los financieros, sino que se requería de otros indicadores de carácter no financiero, tales como indicadores que miden la relación con los clientes, la innovación, la tecnología utilizada en operaciones con clientes, proveedores y en los procesos de fabricación, el clima organizacional y el empoderamiento de los trabajadores. Ante este nuevo escenario se hizo necesario el surgimiento de herramientas que pudieran informar a los gerentes sobre el desempeño de sus ne- 
gocios, a partir de indicadores no financieros, los cuales en la actualidad describen y comunican la estrategia de la organización (Kaplan y Norton, 2000).

Por otra parte, el cuadro de mando integral es una herramienta gerencial estratégica que vincula el desempeño y la estrategia a través de un tablero de mando en el cual se presentan indicadores de carácter financiero como no financiero. Kaplan y Norton (2000) consideran que dicho cuadro provee a los gerentes de la información que necesitan para lograr un negocio exitoso y competitivo.

Sim y Koh (2001) determinaron que las empresas que cuentan con medidas de desempeño no financieras, que contribuyen a monitorear la implementación y ejecución de las estrategias vinculadas con las metas de la organización, presentan un mejor desempeño que aquéllas que no cuentan con este conjunto de medidas.

En cambio Ittner y Larcker (2003) encontraron que la simple inclusión de indicadores no financieros no es suficiente para garantizar el éxito de una organización porque es necesario relacionarlo con las metas estratégicas de la misma. Estos autores descubrieron que muchas empresas estaban incluyendo como indicadores no financieros los que medían fidelidad de los clientes, satisfacción de los empleados, entre otros, por considerar que afectaban la creación de valor. Sin embargo, Ittner y Larcker no pudieron relacionar estos indicadores con las metas estratégicas o establecer una conexión entre las actividades relacionadas con dichos indicadores no financieros y las utilidades alcanzadas en un periodo. Para Tariq (2007) la perdida de dicha conexión ocasionaba que las organizaciones efectuaran malas inversiones o premiaran la ineficacia de los gerentes.

Otros estudios, como el de Jusoh et al. (2006), examinan los aspectos de alineamiento de las medidas con la estrategia y categorizan a las compañías de acuerdo con el abordaje de las estrategias propuesto por Miles y Snow (1978), quienes analizan las medidas de desempeño de las compañías. En su estudio encuestaron a 120 gerentes de empresas manufactureras en Malasia; el punto central era determinar la suplantación de indicadores financieros por no financieros, en especial, la satisfacción del cliente, los procesos internos y el aprendizaje y crecimiento. Los resultados obtenidos indicaron el uso de medidas de clientes, así como de aprendizaje y crecimiento, las cuales al ser combinadas con las estrategias impactaban significativa y positivamente el desempeño de la organización. Indudablemente, las medidas financieras producen un impacto significativo y positivo en el des- 
empeño de las compañías que son categorizadas como usuarias primarias de una estrategia a la defensiva.

\section{Herramientas gerenciales en el proceso de toma de decisiones en la organización}

Debido a que los gerentes de las organizaciones de las Mipyme de Cali se enfrentan al nuevo panorama de manejar una mayor cantidad de información para tomar decisiones acertadas y oportunas, se hace necesario el empleo de herramientas gerenciales que faciliten este proceso.

Según Drucker (1995) desde que aparecieron las primeras herramientas para el procesamiento de datos, hace treinta o cuarenta años, los gerentes han sobrestimado o subestimado el uso de la información para la toma de decisiones en las organizaciones. La sobreestimación se da cuando los gerentes empiezan a crear modelos gerenciales que operados por una computadora podrían tomar decisiones y hasta gerenciar gran parte del negocio. Esto subestimaría la gestión de los gerentes porque se podría pensar que lo que ellos hacen sería remplazado por estos modelos y las computadoras.

De igual forma, Drucker considera que las nuevas herramientas traen cambios que no sólo afectan la forma cómo se realiza la gestión, sino que también la forma cómo los negocios son vistos: generadores de recursos, eslabones de cadenas económicas, órganos de la sociedad para la creación de riqueza, innovadoras y a la vez criaturas de un ambiente material.

De esta manera, las herramientas gerenciales permiten un cambio de visión frente a la organización; además, apoyan a los gerentes en la toma de decisiones oportunas y adecuadas que permiten el uso eficiente de los recursos.

Por otra parte, la modificación en la estructura de $\operatorname{costos}^{4}$ en la era de la información llevó a que las empresas buscaran la mejor manera de asignar los costos indirectos de fabricación a sus productos. De este modo, la nueva forma que permitiría asignar los costos a los productos debería considerar el factor de cambio en la importancia del costo indirecto de fabricación versus la mano de obra directa.

\footnotetext{
${ }^{4}$ El cambio en la estructura de costos consistió en que la participación de la mano de obra como elemento del costo de un producto se hizo menos significativa en comparación con los costos indirectos de fabricación que en los últimos años llegan a ser casi el 60-80\% de los costos totales de un producto.
} 
En la década de los ochenta, la situación que vivió la General Electric en cuanto al aumento de los costos indirectos de fabricación, como parte del costo de un producto, ya era generalizada para muchas empresas americanas. Consecuencia de lo anterior, los gerentes de estas empresas comenzaron a pensar en alguna forma que les permitiera asignar adecuadamente los costos indirectos a los productos, de tal forma que los productos quedaran costeados de la mejor manera; es así que aparece el costeo basado en actividades o costos (ABC).

Asimismo, Drucker (1995) plantea que la determinación de costos por actividad representa una definición distinta del concepto tradicional en el que el costo total de manufactura se limitaba a la información que era suministrada por el área de producción limitada a tres medidas fundamentales: materiales, mano de obra y costos indirectos de fabricación. Sin embargo, con el advenimiento de la nueva manera de calcular los costos, se puede costear a través del ciclo de vida del producto como consecuencia de que son las actividades y no los productos o servicios finales los que determinan los costos diferentes en comparación con el costeo tradicional. De esta manera, el método de costo por actividad muestra (o por lo menos trata de mostrar) el impacto que los cambios en costos y rendimiento de cada actividad ejercen sobre los resultados de la empresa.

Otra de las herramientas gerenciales que tuvieron su origen a finales del siglo pasado es la teoría de las estricciones (TOC). Goldratt, creador de esta herramienta gerencial, considera que la meta de todo negocio es ganar dinero, por lo cual todas las decisiones deben estar orientadas a este fin. Este autor (1997) establece que existe una ganancia (throughput), que es igual a las ventas menos el costo de los materiales directos. Él presenta un modelo de decisión que consta de cinco pasos: 1) identificar la restricción, 2) aprovechar las restricciones del sistema, 3) subordinar todo el sistema a la restricción, 4) elevar la restricción y 5) identificar una nueva restricción.

Además, el creador de esta teoría complementa su modelo de decisión con el sistema tambor-pulmón-cuerda para implementar la Toc en una típica operación de producción. Este sistema se establece una vez que se ha identificado el recurso cuello de botella, dado que con su implementación se pretende que los recursos no cuello de botella estén en consonancia con la capacidad del primer recurso, lo que significa que todo el sistema al interior del sistema debe estar subordinado al ritmo de este recurso (tambor). Igualmente, el recurso cuello de botella nunca debe parar en su proceso productivo por falta de materia prima o producto en proceso; por lo 
tanto, siempre debe existir un inventario (pulmón) que garantice su permanente desempeño; por su parte, la función de la cuerda es la de controlar las operaciones al inicio del proceso, en cuanto que los equipos no cuello de botella se alineen con el recurso no restrictivo.

Por otro lado, la última herramienta gerencial que se aborda en este trabajo es el cuadro de mando integral (CMI) o balanced scorecard (BSC), creada por Kaplan y Norton a principio de la década de los noventa. Según estos autores (2001), la finalidad de CMI es la de monitorear la ejecución e implementación de las estrategias identificadas para lo cual cuenta con medidas, inductores e iniciativas. Cabe señalar que en el CMI, las estrategias se ubican en cuatro perspectivas: la financiera, la de clientes, la de los procesos internos, la de aprendizaje y crecimiento; todas ellas conforman el mapa estratégico que se convierte en el medio por el cual se identifican las estrategias que deberán ser implementadas para alcanzar el éxito deseado.

Una vez que se han identificado los objetivos estratégicos que aparecen en el mapa estratégico, lo cual indica que éste sea considerado como la representación gráfica y conceptual de las estrategias de una organización, se llevan al tablero de mando -que es un elemento que permite a los gerentes conocer el logro de los objetivos a través del monitoreo de los indicadores-iniciativas estratégicas e inductores.

Finalmente, algunas investigaciones concluyen que muchas compañías han encontrado en el cuadro de mando integral, una herramienta útil para focalizar y mantener sus esfuerzos de mejoramiento continuo (Brewer, 2002; Gumbus y Lyron, 2002; Kershaw y Kershaw, 2001).

\section{Hipótesis}

Con base en el marco teórico expuesto y en concordancia con lo expuesto en la formulación del problema y los objetivos se plantean las siguientes hipótesis:

$H_{I}$ : La única fuente de información e indicadores para la toma de decisión utilizada por los gerentes de la Mipyme está representada en los datos y fuentes de carácter financiero.

$H_{2}$ : Existe desconocimiento por parte de los gerentes de las Mipyme en torno a nuevas herramientas gerenciales que contribuyan con el proceso de toma de decisión. 
Este trabajo se centra en los resultados concernientes a las dos hipótesis planteadas. De esta manera, se estudiarán dos temáticas: las fuentes de información, que son indicadores que utilizan los gerentes de las Mipyme para tomar decisiones al interior de la organización que lideran; e identificar si dichos gerentes conocen y usan algunas de las herramientas gerenciales propuestas.

\section{Metodología}

\section{Método y muestra}

Esta investigación emplea métodos cualitativos y cuantitativos para el procesamiento e interpretación de los resultados obtenidos. El tipo de estudio es de carácter inductivo y descriptivo. Para determinar el tamaño de la muestra se utilizó el muestreo aleatorio simple, el cual consiste en seleccionar una muestra de $\mathrm{n}$ elementos uno a uno de una población $N$, de tal forma que cada unidad poblacional tenga una probabilidad idéntica y conocida de pertenecer a la muestra. Aquí cada elemento se selecciona en forma independiente de los demás; su extracción se realiza mediante un proceso aleatorio a partir de un marco de muestreo. El muestreo aleatorio simple no sólo asegura una muestra representativa, sino que también produce una estimación de la cantidad de una población y una especificación de la precisión.

Para calcular el tamaño de la muestra, en esta investigación se utilizó la fórmula que se basa en la metodología de muestreo aleatorio simple para proporciones (Anderson, 1999):

$$
n_{0}=\frac{Z_{1-\alpha}^{2} \cdot P \cdot P \cdot Q}{\delta^{2}}
$$

De acuerdo con el Censo Económico de Cali, Yumbo 2005, existen 53.417 micro, pequeñas y medianas empresas, lo que correspondería al total de la población $\mathrm{N}$. Se tomó como nivel de confianza $95 \%$ y un error de muestreo de $0,05 . \delta=0,05$. En la tabla de distribución normal se busca el valor de $\mathrm{Z}$ que corresponde a un nivel de confianza del 95\%; este número corresponde a 1,6448. Como no se tenían estimaciones adelantadas de las proporciones se utilizó asignación de mínima varianza, es decir $\hat{P}=0,50$ y $\hat{Q}=0,50$. 
Reemplazando los valores en la fórmula:

$$
n_{0}=\frac{(1,6448)^{2} \cdot(0,5)(0,5)}{(0,05)^{2}}=270,4
$$

Este valor se aproxima a 270; éste es el tamaño de la muestra que se debe tomar. La idea inicial era distribuir este número proporcionalmente en los diferentes tamaños de empresas (micro, pequeña y mediana); sin embargo, no se logró encuestar el número suficiente de medianas empresas (47 empresas), por lo que las encuestas que faltaron se distribuyeron en las micro y pequeñas empresas. De esta manera, la distribución final de las encuestas se presenta en el siguiente cuadro ${ }^{5}$ :

\section{Cuadro 1}

Distribución de las encuestas por tipo de empresa

\begin{tabular}{ccc}
\hline Tipo de empresa & No. de encuestas & $\%$ \\
\hline Micro & 111 & $41,1 \%$ \\
Pequeña & 112 & $41,5 \%$ \\
Mediana & 47 & $17,4 \%$ \\
\hline Total & 270 & $100 \%$
\end{tabular}

Dentro de la encuesta se indagó por el tipo de actividad económica al que se dedican las empresas. Para el total de las encuestas realizadas (270), el $42 \%$ de las empresas se dedica a actividades comerciales, seguida por los servicios con $32 \%$ y, por último, un $26 \%$ en actividades industriales.

\section{Cuadro 2}

\section{Actividades económicas por tipo de empresa}

\begin{tabular}{ccccc}
\hline \multirow{2}{*}{$\begin{array}{c}\text { Tamaño } \\
\text { de empresa }\end{array}$} & \multicolumn{2}{c}{ Tipo de empresa por actividad económica } & \multirow{2}{*}{ Total } \\
\cline { 2 - 4 } & Comercial & Servicios & Industrial & \\
\hline Microempresa & 54 & 29 & 28 & 111 \\
Pequeña & 37 & 45 & 30 & 112 \\
Mediana & 21 & 13 & 13 & 47 \\
\hline Total & 112 & 87 & 71 & 270
\end{tabular}

\footnotetext{
${ }^{5}$ En Colombia, según la Ley para el Fomento de la Micro, Pequeña y Mediana Empresa, Ley 590 de 2000 , las Mipyme se clasifican así: Microempresa: personal no superior a 10 trabajadores; activos totales inferiores a 501 salarios mínimos legales vigentes. Pequeña empresa: personal entre 11 y 50 trabajadores. activos totales mayores a 501 y menores a 5.001 smlv. Mediana: personal entre 51 y 200 trabajadores; activos totales entre 5.001 y 15.000 smlv. Para esta investigación el tamaño de las empresas se tomó de acuerdo con el valor de los activos.
} 


\section{Delimitación del trabajo}

El estudio se delimita dentro de los siguientes aspectos:

- Geográfica: la investigación se realizó en el área metropolitana de Cali.

- Población objetivo: micro, pequeñas y medianas empresas de Cali.

- Temporal: las encuestas se aplicaron durante los meses de octubre a diciembre de 2006 y el primer semestre de 2008.

- Tamaño de la muestra: 270 empresas.

- Los indicadores financieros y no financieros como las herramientas gerenciales corresponden a las propuestas para este trabajo a pesar de que existen otros indicadores y herramientas diferentes a los propuestos.

- Este trabajo se limita a presentar los aspectos descriptivos de la investigación.

\section{Análisis de datos y resultados}

De acuerdo con el problema de investigación y con las hipótesis planteadas, el resultado de las encuestas proporcionó resultados interesantes con respecto a las fuentes de información, indicadores y herramientas que utilizan los gerentes de las Mipyme en Cali. A continuación se exponen los principales resultados.

\section{Fuentes de información para la toma de decisiones e indicadores financieros}

\section{- Fuentes de Información}

Conforme a los resultados obtenidos en las entrevistas realizadas se puede constatar que los gerentes de las microempresas $(54.10 \%)$ y pequeñas (48.2\%) se apoyan en los datos financieros básicos que se derivan del Balance General, Estado de Resultados y Flujo de Caja. Los anteriores porcentajes corroboran, parcialmente, la hipótesis 1 de la investigación para este tipo de empresas, que afirma que la única fuente de información para la toma de decisión está representada en los datos y fuentes de carácter financiera. Por el contrario, es más evidente que los gerentes de las medianas empresas toman sus decisiones a partir de la confluencia tanto de datos financieros como no financieros $(63.8 \%)$. Los gerentes de las pequeñas empresas $(47.30 \%)$ casi en igual porcentaje a los resultados obtenidos en la opción anterior, toman decisiones a partir de fuentes tanto financieras como no financie- 
ras. Contrariamente, los gerentes de las micro (36\%) son los que menos toman decisiones a partir de fuentes financieras y no financieras.

\section{- Indicadores financieros-no financieros}

En cuanto a indicadores financieros conforme a los resultados obtenidos se puede constatar que los gerentes se interesan por la continuidad del negocio; por tanto, en sus análisis financieros consideran los indicadores de rentabilidad como importantes. Así, los mayores porcentajes en las respuestas dadas por los gerentes hacen referencia a involucrar indicadores de rentabilidad, los cuales resultan relevantes porque permiten que los gerentes informen acerca de la rentabilidad generada por los capitales invertidos; esto es, cuánto rindieron las inversiones y, en consecuencia, cuál es el grado de éxito económico que al final garantiza la continuidad del negocio.

En las microempresas, la combinación de indicadores de rentabilidad y endeudamiento (40.5\%) es la que más se considera para la toma de decisión financiera; esto puede indicar que los gerentes, al preocuparse por la rentabilidad, les inquieta su continuidad en el largo plazo y el nivel de endeudamiento, pues de este último depende que sus proveedores les vendan a crédito los productos o servicios que requieren para el desarrollo de su objeto social. En las pequeñas (44.6\%) y medianas $(46.8 \%)$ empresas las decisiones financieras se realizan tomando en cuenta la información que suministran, la combinación de indicadores de rentabilidad y liquidez; al igual que en las microempresas, esto indica una preocupación por la continuidad a largo plazo; además de considerar la liquidez, que es importante, el dato de la solvencia financiera para realizar las actividades operacionales es otro elemento necesario.

Asimismo, se constata que a los gerentes les preocupa conocer la liquidez de la empresa para considerar si hay solvencia financiera, las probabilidades de pagar a los proveedores y acreedores son altas. Esto es importante porque de no existir los recursos financieros suficientes, no se podrían obtener los servicios o materias primas necesarias para el desarrollo del objeto social, con lo cual se pondría en peligro la continuidad del negocio. Tener liquidez garantiza el pago de dividendos a los accionistas (para los casos que aplique) y el pago a los empleados de sus prestaciones sociales. En cuanto a la combinación de indicadores de liquidez y endeudamiento, éstos son de interés para algunos gerentes, pero en un menor porcentaje, si se compara con las anteriores combinaciones presentadas. 
Finalmente, se constata el bajo uso del Valor Económico Agregado (EVA) como indicador financiero para toma de decisiones. Los gerentes de las microempresas desconocen totalmente su existencia y, por tanto, no lo utilizan; en las pequeñas empresas sólo el $0.9 \%$ dice utilizarlo y en las medianas el $6.4 \%$ de los gerentes lo calculan para fines de toma de decisión. Esto nuevamente va unido con el grado de complejidad del negocio; en este caso, adicionaríamos otra variable como la capacitación y formación de los gerentes; es conocido que quienes estén más capacitados tendrán mayor conocimiento sobre herramientas e indicadores que les facilitarán el proceso de toma de decisión.

Por lo que respecta a los indicadores no financieros, de conformidad con los resultados obtenidos para los tres tipos de empresas se encontró que el indicador no financiero que prevalece para fines de toma de decisión es el de la atención a clientes; esto va muy acorde con las tendencias mundiales, pues la relación con los clientes se constituye en un indicador importante si tomamos en cuenta que la continuidad de un negocio depende de la fidelidad, satisfacción y de ganar nuevos clientes.

Los resultados observados permiten concluir que los gerentes de las microempresas $(56.8 \%)$ le dan una gran relevancia a todo aquello que le permita conocer el nivel de satisfacción de los clientes, lo cual se puede conocer a través de la respuesta que se obtenga al calificar la atención a clientes. Preocupa que en este tipo de negocios sea bajo el interés por aspectos como capacitación del personal (6.3\%) y nuevas tecnologías implementadas $(8.1 \%)$. Con respecto a la innovación en los procesos de producción (11.7\%) este tipo de empresas tiene el mayor porcentaje en relación con las otras; esto es consecuencia de la fragilidad que presentan las microempresas para mantenerse vigentes en el mercado en comparación con las pequeñas y las medianas empresas, lo cual estimula la innovación en ellas. Asimismo, el $13.5 \%$ de las microempresas dicen tomar en cuenta todos los activos intangibles propuestos para la investigación, los cuales dan origen a indicadores no financieros en la toma de decisión. En cuanto a otros indicadores no financieros diferentes a los propuestos, la mayoría de respuestas se centra en la mejoría de los procesos de producción que han permitido disminuir la intervención de terceros en él, con ello se corrobora el resultado obtenido en la pregunta sobre innovación en los procesos.

Un comportamiento similar se observa en las pequeñas y medianas empresas en las cuales la atención a clientes (39.3\% y 38.3\%, respectivamente) es importante en la 
toma de decisión. Los aspectos relacionados con capacitación, nuevas tecnologías implementadas e innovación en los procesos de producción no alcanzaron porcentajes importantes para ambos tipos de empresas. No obstante, se observa que en las medianas empresas aspectos como capacitación del personal (14.9\%) y nuevas tecnologías implementadas (10.6\%) tienen los porcentajes más altos de respuesta en relación con las micro y pequeñas empresas.

En relación con otros indicadores no financieros, identificados por los gerentes de las pequeñas empresas (5.4\%), se hace referencia a indicadores que les permiten medir el grado de participación en el mercado y la oportunidad en la negociación. Algunos gerentes de pequeñas empresas dijeron no tomar en cuenta indicadores no financieros dado que las decisiones se toman con base en los resultados observados en los estados financieros y en el avance de ciertas actividades. Por su parte, las medianas empresas consideran indicadores no financieros que miden el mejoramiento de procesos productivos, administrativos y de clima laboral, así como sistema de gestión de calidad-Iso.

Conforme a lo observado se puede constatar que la tendencia evidenciada en los tres tipos de empresas es que los gerentes suelen utilizar como principal fuente de información los estados financieros tradicionales que para este estudio fueron seleccionados: Balance General, Estado de Resultados, Flujo de Caja. No obstante, en las empresas medianas se observa que la mayor parte de los gerentes en comparación con los de las pequeñas y micro suele combinar información financiera con no financiera para la toma de decisión. También se comprueba que no es frecuente utilizar diferentes estados financieros a los tradicionales para fines de toma de decisión en los tres tipos de empresas.

\section{Herramientas gerenciales para la toma de decisiones}

- Herramientas gerenciales (de las propuestas) más conocidas por los gerentes

Las Mipyme deben contar con herramientas gerenciales que les permitan conocer su estrategia además de que facilite el proceso y seguimiento de la implementación y ejecución de la misma. Cabe señalar que para el seguimiento de esta última tarea es necesario que exista algún mecanismo de medición que permita valorar el logro de la estrategia ejecutada. 
La necesidad de conocer herramientas gerenciales que ayuden a las Mipyme a su sobrevivencia y éxito es innegable. De acuerdo con los resultados obtenidos en relación con la pregunta del conocimiento de algunas de las herramientas gerenciales propuestas como cuadro de mando integral, costos ABC, teoría de las restricciones (ТОC), se concluye que los gerentes de las micro en un alto porcentaje (55\%) en comparación con el porcentaje de respuestas dadas por los gerentes de pequeñas (35.7\%) y medianas empresas $(27.7 \%)$ no conocen ninguna de las herramientas gerenciales de las propuestas para el estudio.

Por otra parte, se observa que de las tres herramientas gerenciales (cuando los gerentes dijeron conocer alguna de ellas) las más conocidas son los costos ABC y el cuadro de mando integral, mientras que la menos conocida es la TOC. Se constata que en bajos porcentajes los gerentes de las pequeñas empresas (30.4\%) dicen conocer todas las herramientas, le siguen los gerentes de las medianas (29.8\%) y por último sólo el $8.10 \%$ de la muestra total de gerentes de microempresas encuestados afirman conocer todas las herramientas propuestas para este trabajo.

Como consecuencia de lo anterior, se confirmaría la hipótesis dos de la investigación que hace referencia al desconocimiento por parte de los gerentes de las Mipyme en torno a nuevas herramientas gerenciales; esto en relación con el alto porcentaje que se obtuvo por parte de los gerentes encuestados en cuanto a no conocer ninguna de las herramientas gerenciales.

- Implementación de alguna(s) de las herramientas propuestas

Con respecto a la pregunta que hace referencia a la implementación de alguna de las herramientas gerenciales mencionadas, las pequeñas $(38.4 \%)$ y medianas $(40.4 \%)$ muestran mayores porcentajes de respuesta con relación a la implementación de dichas herramientas, a diferencia de las microempresas (18\%) que evidenció los menores porcentajes de implementación.

Las micro (82\%) presentan el mayor porcentaje de no haber implementado ningún tipo de herramienta gerencial frente a los porcentajes obtenidos por las pequeñas $(61.6 \%)$ y las medianas (56.6\%); asimismo, se observó que para todos los casos los porcentajes de no implementación son altos.

Las herramientas gerenciales más implementadas e identificadas por los gerentes encuestados son los costos ABC y el cuadro de mando integral. Es importante acla- 
rar que una de las herramientas mencionadas por los gerentes y que ha sido implementada es el Enterprise Resource Planning (ERP) como un sistema de planeación de los recursos de la empresa.

- Identificación del motivo que ha impedido implementar alguna de las herramientas gerenciales

Este aspecto está relacionado con el anterior y evidencia que los gerentes de las Mipymes no conocen y, por lo tanto, no implementan las herramientas gerenciales porque las desconocen, lo que indicaría una ausencia de capacitación sobre nuevas técnicas y la falta de estrategias encaminadas a mejorar y fortalecer las capacidades, así como habilidades de los empleados — por consiguiente de los gerentessobre estos asuntos gerenciales.

El $47.2 \%$ de los gerentes de las microempresas encuestadas afirman que no utilizan las herramientas por desconocimiento, el $22.2 \%$ porque no tienen tiempo para dedicarle a la implementación y el $13.9 \%$ porque no tiene dinero para este tipo de asuntos.

Con respecto a los gerentes de las pequeñas empresas, el 35\% afirma que desconoce las herramientas, el $20.4 \%$ no le ve la pertinencia y el $17.5 \%$ no tiene tiempo para dedicarle a la implementación.

Por otro lado, en las medianas empresas el $29.5 \%$ de los gerentes dijeron desconocer las herramientas y afirmó que no tener tiempo para dedicarse a su implementación. Es interesante observar que a medida que aumenta el tamaño de la empresa el porcentaje de respuesta que afirma que desconoce las herramientas disminuye, lo que podría indicar que a medida que aumenta el tamaño de la empresa existe un mayor conocimiento de las herramientas gerenciales modernas. Intuitivamente podemos suponer que los gerentes de las medianas y pequeñas empresas están mejor preparados que los gerentes de las micro, lo cual que se confirmará más adelante.

- Principios en los que se basa el modelo de decisión gerencial de las Mipyme

El modelo de decisión gerencial de las Mipyme se basa en principios generales básicos de la contabilidad, finanzas y administración tradicional. En las microem- 
presas, la respuesta frente a esta opción fue de $76.6 \%$, en las pequeñas de $71.4 \%$ y en las medianas de $70.2 \%$.

En las microempresas, el $8.1 \%$ utiliza los principios generales de la gestión y el costo basado en actividades; tan sólo el $2.7 \%$ afirmó utilizar principios generales del cuadro de mando integral. En las pequeñas empresas el $16.1 \%$ toma sus decisiones con base en los principios generales de la gestión y el costo basado en actividades y el 5.4\% afirmó que utiliza los principios de cuadro de mando integral.

En las medianas empresas el porcentaje de utilización de los principios generales de la gestión y el costo basado en actividades es del 17\%; mientras que el de utilización del cuadro de mando integral es de 6.4\%. Es interesante observar que ningún gerente encuestado afirmó que su modelo de toma de decisión se basa en los principios generales de la teoría de restricciones.

Los resultados obtenidos permiten concluir que existe un bajo nivel de conocimiento por parte de los gerentes de las Mipyme en relación con estas herramientas (cuadro de mando integral, teoría de restricciones y costo basado en actividades) para la toma de decisiones al interior de la organización.

- Asistencia de los gerentes a seminarios de actualización relacionados con nuevas técnicas gerenciales

Con respecto a la asistencia de los gerentes a seminarios de actualización sobre técnicas gerenciales es evidente la diferencia que se presenta en el tamaño de las empresas; a medida que aumenta el tamaño de las empresas, aumenta también la asistencia a seminarios de actualización, lo que indica una relación positiva entre estas dos variables. Para las microempresas, el $30.6 \%$ asiste eventualmente a este tipo de seminarios, las pequeñas y medianas empresas asisten por lo menos una vez al año (33.9\% y 40.4\%, respectivamente) lo que muestra la importancia de la actualización sobre técnicas gerenciales para las pequeñas y medianas empresas.

Lo anterior lleva a concluir que sí existe un interés por parte de estas unidades de negocio con respecto a la capacitación; sin embargo, dado los porcentajes obtenidos, se podría afirmar que es muy moderado con tendencia a ser bajo. Finalmente, se constata que el $21.6 \%$ de los gerentes de las microempresas nunca asisten a cursos o seminarios de actualización en técnicas gerenciales; los gerentes de las pequeñas en un $8.9 \%$ y los de las medianas $0 \%$, lo que indica que los gerentes de 
las medianas sí asisten a cursos de actualización; en este tipo de empresas no se concibe una falta total de capacitación por parte de los gerentes.

\section{Conclusiones}

A partir del análisis de los resultados de las encuestas aplicadas a las Mipyme en la ciudad de Cali, se concluye que la hipótesis uno, en la que se afirma que la única fuente de información para toma de decisión está representada en los datos financieros, no se puede corroborar porque los gerentes en relación con cada tamaño de empresa prefiere y utiliza diferentes indicadores tanto financieros como no financieros a la hora de tomar decisiones.

En las microempresas, los indicadores que más se toman en cuenta son los de rentabilidad y endeudamiento; en cuanto a las pequeñas y medianas empresas, las decisiones financieras se toman de acuerdo con los indicadores de rentabilidad y liquidez.

En cuanto a indicadores no financieros la tendencia de las pyme es preferir los que miden la atención a clientes, capacitación de personal e innovación en los procesos de producción, lo que resulta distinto en relación con lo que sucede con las microempresas que le prestan poca atención a este tipo de indicadores no financieros, con excepción de atención a clientes que, al igual que en las pyme, se observa preferencia por conocer su impacto sobre clientes.

Los gerentes de las micro y pequeñas empresas toman sus decisiones con base en los datos que le suministran fuentes de información como los estados financieros básicos: Balance General, Estados de Resultados, Flujo de Caja, entre otros; en cambio sucede lo contrario en las medianas empresas, pues las decisiones no sólo se toman con relación a los datos que suministran los estados financieros básicos, sino que también se consideran otros tipos de informes con información no financiera relacionados con mercado, clientes, recurso humano e innovación.

De acuerdo con la hipótesis 2, que afirma que existe desconocimiento por parte de los gerentes de las Mipymes en torno a nuevas herramientas gerenciales, ésta se corrobora de manera preliminar debido a que un gran porcentaje de los gerentes de las microempresas, pequeñas y medianas empresas afirman no conocer las herramientas gerenciales preguntadas. 
Los gerentes de las micro y pequeñas empresas, según los resultados de las encuestas, no emplean las herramientas gerenciales propuestas para esta investigación porque simplemente no las conocen; esto indica una ausencia de capacitación por parte de ellos sobre nuevas técnicas gerenciales, lo cual se confirma cuando se pregunta con qué frecuencia se asiste a seminarios de actualización. Los resultados de las encuestas muestran que los gerentes de las empresas medianas, en general, por lo menos una vez al año asisten algún tipo de curso. En ese sentido, los que menos asisten a cursos de capacitación son los gerentes de las micros y, contrariamente, los gerentes de las medianas empresas son los que más participan en cursos, o en alguna capacitación, una vez al año.

A pesar de que en un alto porcentaje los gerentes de las Mipyme manifestaron no conocer las herramientas gerenciales, los que contestaron conocer alguna coincidieron en señalar a los costos $\mathrm{ABC}$ y al cuadro de mando integral; en cuanto a teoría de las restricciones, todos los gerentes afirmaron no haberla implementado, además un bajo porcentaje indicó conocerla.

\section{Referencias}

Anderson, David R. (1999). Estadística para administración y economía. México.

Brewer, P. (2002). "Putting strategy into the balanced scorecard". Strategy Finance, January, 44-52.

Choo, Wei Chun (1999). "La organización inteligente". Oxford: Oxford University Press, 346.

Departamento Administrativo Nacional de Estadística (DANe) (2005). Censo Económico Cali, Yumbo, 2005.

Documento Conpes 3484 (2007). Política nacional para la transformación productiva y la Promoción de las micro, pequeñas y medianas empresas: Un esfuerzo público-privado.

Documento Conpes 3424 (2006). La banca de las oportunidades. Una política para promover el acceso al crédito y a los demás servicios financieros buscando equidad social. 
Drucker, Peter (1995). "Su visión sobre la administración, la organización basada en la información, la economía, la sociedad". Managing in a time of great change. New York: Truman Talley Books/Dutton.

(1993). Post-Capitalist Society. Nueva York: Harpenter Collins.

Goldratt, Eliyahu M. and Cox, Jeff (1997). A Meta. 35ª ed., São Paulo: Educator.

Gumbus, A. and Lyron, B. (2002). "The balanced scorecard at Philips electronics". Strategic Finance, 45-49.

Jusoh, R., Ibrahim, D. and Zainuddin, Y. (2006). "Assessing the alignment between business strategy and use of multiple performance measures using interaction approach". The business review, Vol 5, No. 1, 51-60.

Ittener, C. and Larcker, D. (2003). "Coming up short on non-financial performance measurement". Harvard Business Review, vol. 81, no. 11, 88-95.

Kaplan, Robert and Norton, David (2001). "Transforming the balanced scorecard from performance measurement to strategic management: Part I and Part II". Accounting Horizons. Vol. 15.

-(2000). Cuadro de mando integral. $2^{\mathrm{a}}$ ed., Barcelona: Gestión 2000, 321.

\& COOPER, Robin (1999). Coste y efecto. Barcelona: Gestión 2000, 390.

Kershaw, R. and Kershaw, S. (2001). "Developing a balanced scorecard to implement strategy at Sta. Elsewhere Hospital". Management Accounting Quarterly, Winter, 28-35.

March, J. G. (1976) A primer on decision making: how decision happen. New York, Free Press.

Miles, R. and Snow, C. (2007). "Organizational Strategy: Structure and Process", Mc Graw Hill, New York, in: Tarique H. Ismail, "Performance evaluation measures in the private sector: Egyptian practice". Managerial Auditing Journal, Vol. 22, No.5, 503-313. 
Simon, H. A. (1993). Administrative Behavior: a Study of decision-making process in, $3^{\mathrm{a}}$ ed., New York.

Sim, K., and C. Koh (2001). "Balanced scorecard: a raising trend in strategic performance measurement". Measuring Business Excellence, Vol. 5, No. 2, $18-26$.

Tariq H., Ismail (2007). "Performance evaluation measures in the prívate sector: egyptian practice". Managament Auditing Journal, Vol 22, No. 5, 503-513. 